\title{
Cosmic Power Generation and Gravity
}

\author{
Greg Poole \\ Industrial Tests Inc., Rocklin, CA, USA \\ Email: greg@indtest.com
}

How to cite this paper: Poole, G. (2019)

Cosmic Power Generation and Gravity. Journal of High Energy Physics, Gravitation and Cosmology, 5, 920-927. https://doi.org/10.4236/jhepgc.2019.53047

Received: May 22, 2019

Accepted: July 14, 2019

Published: July 17, 2019

Copyright $\odot 2019$ by author(s) and Scientific Research Publishing Inc. This work is licensed under the Creative Commons Attribution International License (CC BY 4.0).

http://creativecommons.org/licenses/by/4.0/

\section{(c) (i) Open Access}

\begin{abstract}
By modeling the Sun as an electrical dynamo, the speed and frequency of the Sun's electromagnetic field is determined. The results confirm the approximate radius of the Sun's inner core 1,220,000 meters and gravity at its surface, $274 \mathrm{~m} / \mathrm{s}^{2}$. The Sun's rotating electromagnetic field radiates to Pluto and beyond. Like gravity, the near magnetic field of a sphere weakens with the inverse square of the distance. The Sun is a constant speed and constant acceleration machine. Like a set speed Ferris wheel, the orbital speed of planets is faster nearer to the Sun and reduces as the distance increases. The Standard Gravitation Parameter $\left(\mathrm{m}^{3} / \mathrm{s}^{2}\right)$ is analogous to accelerating cubic volume $\left(\mathrm{m}^{3} / \mathrm{s}^{2}\right)$ of the solar system. The planets are being pushed away by a centrifugal force from the constant acceleration of the Sun's magnetic field, while at the same time being pulled in by the force of the Sun's centripetal acceleration. Planetary orbits are the result of this balancing of forces. Gravity is a centripetal acceleration derived from a rotating electromagnetic field. Gravity is derived from an electromagnetic field which means it is not a force. There are only three forces in the universe: electromagnetic, strong nuclear and weak nuclear.
\end{abstract}

\section{Keywords}

Centripetal Acceleration, Gravity (g), Orbital Velocity, Dynamo, Standard Gravitational Parameter

\section{Introduction}

Sir Joseph Larmor first proposed the Sun as a self-exciting dynamo at the 7th meeting of the British Association for the Advancement of Science on September 9th, 1919. His seminal paper established the cosmic dynamo theory for the first time. The focus of his paper was to explain the Sun's permanent magnetic field, but he introduced the Earth's magnetic field as well [1].

Larmor was raised in Magherhall County Antrim, and went to school at the 
Royal Belfast Academical Institution and Queen's College in Belfast. He was senior wrangler in the Mathematics Tripos at Cambridge. J.J. Thomson was placed second in the competition. Larmor was the Lucasian Professor of Mathematics following George Stokes, and was knighted in 1909. Larmor worked with James Clerk Maxwell, J.J. Thomson and Oliver Heaviside when electricity was just coming of age following the work of Michael Faraday. Larmor's book, Aether and Matter, was considered revolutionary and is in my personal collection. Along with Lorentz and those mentioned, Larmor is considered an early founder of the principles of electromagnetism [2].

A century after Sir Joseph Larmor published his revolutionary thought on the Sun's magnetic field, my paper moves Sir Joseph Larmor's dynamo theory forward and models the Sun's inner core as a rotating armature using a standard motor speed equation. It is thought that the Sun acts like the prime mover, generator and power transmitter in the solar system, and as such self-excites, thus creating its own electrical frequency. Using known distances and orbital velocities of the planets, I look to leverage a common basis in which to establish the speed of the Sun's rotating magnetic field, which will be used to determine frequency and confirm other physical parameters of the Sun. In the calculations to follow, I have assumed the inner core of the Sun to be shaped as a near perfect sphere. The shape of a transmitter or receiver antenna dictates the rate of the decreasing electromagnetic field as the distance increases. The magnetic near field of a sphere decreases as an inverse square of the distance or $1 / r^{2}$, which is the same relationship as gravity $(g)$.

\section{Calculate the Standard Gravitation Parameter of the Planets}

The orbital equation is expressed as:

$$
r=\frac{h^{2}}{\mu}[3]
$$

where $h=r \nu$ is the specific angular momentum of an orbiting body and $\mu$ is the standard gravitational parameter, which then converts to:

$$
\mu=r v^{2}
$$

where $r$ is the orbital radius and $v$ is the orbital speed.

The standard gravitational parameters for each of the 8 planets and Pluto are shown in Table 1.

\section{Calculate Orbital Velocity of the Sun's Electromagnetic Field}

I now calculate the orbital velocity of the Sun's electromagnetic field.

Since,

$$
\mu=r v^{2}
$$


Table 1. Standard gravitational parameters of different planets and Pluto.

\begin{tabular}{cccc}
\hline Planet & Orbital Velocity $(\mathrm{m} / \mathrm{s})$ & Distance to Sun & $\mu=r v^{2}$ \\
\hline Mercury & 47,400 & $5.79 \times 10^{10}$ & $1.30 \times 10^{20}$ \\
Venus & 35,000 & $1.08 \times 10^{11}$ & $1.32 \times 10^{20}$ \\
Earth & 29,800 & $1.50 \times 10^{11}$ & $1.33 \times 10^{20}$ \\
Mars & 24,000 & $2.28 \times 10^{11}$ & $1.31 \times 10^{20}$ \\
Jupiter & 13,000 & $7.78 \times 10^{11}$ & $1.31 \times 10^{20}$ \\
Saturn & 9700 & $1.43 \times 10^{12}$ & $1.35 \times 10^{20}$ \\
Uranus & 6800 & $2.87 \times 10^{12}$ & $1.33 \times 10^{20}$ \\
Neptune & 5400 & $4.50 \times 10^{12}$ & $1.31 \times 10^{20}$ \\
Pluto & 4700 & $5.91 \times 10^{12}$ & $1.30 \times 10^{20}$ \\
& & Average & $1.32 \times 10^{20}$ \\
\hline
\end{tabular}

$$
v=\sqrt{\frac{\mu}{r}}
$$

Adding the following values into the above equation:

Inner core radius $r=6.96 \times 10^{8} \mathrm{~m} \times 0.20=1.392 \times 10^{8} \mathrm{~m} \quad$ [4]

I calculated the inner core radius based on the estimate that the core is 0.2 times the radius of the Sun.

Standard Gravitation Parameter $\mu=1.32 \times 10^{20} \mathrm{sec}^{2}$

Inserting the values results in:

$$
v=9.66389 \times 10^{5} \mathrm{~m}^{2} / \mathrm{sec}
$$

Since I am using a unitless equation, I express the average orbital speed of the inner core in the customary units of $9.66 \times 10^{5} \mathrm{~m} / \mathrm{s}$, which reflects the same units of the individual planets' orbital speed. The electromagnetic field orbits the edge of the inner core and radiates outwards. Effectively, orbital velocity is what is commonly called synchronous speed of the electromagnetic field. From a standpoint of motor slip this makes sense as an armature in a motor will run slightly behind the synchronous speed of the electromagnetic field. This difference in speed is called slip. The orbital speed or speed of the magnetic field is faster than that of the Sun's inner core. When slip is $0 \%$, or no load, the armature and field speeds are the same speed.

\section{Calculate RPM of Sun's Inner Core}

The Linear Velocity to RPM formula is:

$$
v=r \times 2 \pi \times \frac{\mathrm{RPM}}{60}
$$

or

$$
v=r \times \mathrm{RPM} \times 0.10472
$$


or

$$
\mathrm{RPM}=\frac{v}{r \times 0.10472}
$$

where:

$$
v=9.66389 \times 10^{5}
$$

Radius of the inner iron core $=1.392 \times 10^{8} \mathrm{~m}$

Inserting the values and the result is $0.066295 \mathrm{RPM}$.

\section{Calculate the Frequency of the Sun's Inner Core}

Calculating the magnetic field speed for an armature of a synchronous machine is dictated by the following equation. The mathematical formula to make this calculation is the number of cycles $(\mathrm{Hz})$ times 60 (for seconds in a minute) times two (for the positive and negative pulses in the cycle) divided by the number of poles. Or, $N=120 f / P$ for typical commercial and industrial machines [6].

$$
N=120 \frac{f}{P}
$$

Or

$$
f=\frac{N \times P}{120 \times p}
$$

where,

$$
\begin{aligned}
& N=0.066295 \mathrm{RPM}, \\
& P=\text { number of poles, or } 2, \\
& p=2 \text { for low frequency AC operation. }
\end{aligned}
$$

Inserting the value for RPM and number of poles, I obtain the Sun's operating frequency

$$
f=0.0011 \mathrm{~Hz} \text {. }
$$

\section{Calculate Centripetal Acceleration $\left(\mathrm{m} / \mathrm{s}^{2}\right)$ of Inner Core}

The centripetal ("center-seeking") acceleration is the motion inwards towards the center of a circle. The acceleration is equal to the square of the velocity, divided by the radius of the circular path.

$$
a=\frac{v^{2}}{r} \quad \text { [7] }
$$

where,

$$
\begin{aligned}
& a=\text { acceleration inward, } \\
& v=\text { velocity, or } 966,389 \mathrm{~m} / \mathrm{s}, \\
& r=\text { radius of inner core, or } 139,200,000 \mathrm{~m} .
\end{aligned}
$$

When we insert these values in the centripetal acceleration equation, we obtain a result of $6709 \mathrm{~m} / \mathrm{s}^{2}$, which represents the gravity at the surface of the Sun's inner core. 


\section{Calculate the Sun's Surface Acceleration Using the Altitude (m/s2) Method}

The acceleration due to gravity at an altitude equation typically estimates the acceleration due to gravity on Earth at a specific altitude above sea level [8]. In our scenario we are using the equation to calculate the specific altitude above the Sun's inner iron core, when an object is at the surface of the Sun. The altitude $(h)$ is then the radius of the Sun less the radius of the inner core.

The acceleration due to gravity by altitude formula computes the approximate acceleration due to gravity on the surface of the Sun based on the altitude. This same equation applies to electromagnetic fields that decreases by the inverse square from a sphere no matter how large [9]. The equation for the acceleration due to gravity based on altitude is:

$$
g_{a l t}=g\left(\frac{r_{e}}{r_{e}}+h\right)^{2}
$$

where:

$g_{\text {alt }}=$ the acceleration due to gravity at a specific altitude,

$r_{e}=$ Mean Radius of the Suns Core $\left(r_{e}\right): 1.392 \times 10^{8} \mathrm{~m}$,

$g=$ Acceleration Due to Gravity at Suns Core $(g): 6709 \mathrm{~m} / \mathrm{s}^{2}$,

$h=$ Altitude above the Iron Core: $6.96 \times 10^{8}-1.392 \times 10^{8}=5.568 \times 10^{8} \mathrm{~m}$.

Inserting the values we obtain a result of $268 \mathrm{~m} / \mathrm{s}^{2}$ or $g$ at the surface of the Sun. This value closely approximates the known value of $274 \mathrm{~m} / \mathrm{s}^{2}$ within $1 \%$.

\section{Calculate Centripetal Acceleration $\left(\mathrm{m} / \mathrm{s}^{2}\right)$ of the Sun's Outer Mantle}

The acceleration is equal to the square of the velocity, divided by the radius of the circular path.

$$
a=\frac{v^{2}}{r}
$$

where,

$$
\begin{aligned}
& a=\text { acceleration inward, } \\
& V=\text { velocity, or } 1997 \mathrm{~m} / \mathrm{s}, \\
& r=\text { radius of Sun, or } 6.96 \times 10^{8} \mathrm{~m} .
\end{aligned}
$$

When we insert these values in our centripetal acceleration equation, we obtain a result of $0.005729 \mathrm{~m} / \mathrm{s}^{2}$. The rotational speed of the Sun's outer mantle is not a significant contributor to the Sun's gravity.

\section{Calculate the Centripetal Acceleration of Planets around the Sun}

I have shown how gravity or small $(g)$ is a centripetal acceleration derived from a rotating electromagnetic field. The "near reactive" magnetic field of a sphere decreases by the square of the inverse distance $\left(1 / r^{2}\right)$, which matches that of gravity. The diameter of the Sun is $1.392 \times 10^{9} \mathrm{~m}$, which is less than half the shortest wa- 
velength or distance to the planet Mercury. The Sun is considered an electromagnetically short antenna [10].

Using the orbital velocities of the planets, which match the orbital velocity of the "near radiative" field, I calculate the centripetal acceleration for each of the planets and compare to Newton's Law of Universal Gravitation (Table 2).

The acceleration is equal to the square of the velocity, divided by the radius of the circular path.

$$
a=\frac{v^{2}}{r}
$$

where,

$$
\begin{aligned}
& a=\text { acceleration inward, } \\
& v=\text { orbital velocity of planet }(\mathrm{m} / \mathrm{s}), \\
& r=\text { radius from Sun }(\mathrm{m}) .
\end{aligned}
$$

\section{Conclusions}

My calculations demonstrate that the rotation of the Sun's armature circuit is the primary source of gravity on the surface of the Sun. Gravity is a centripetal acceleration derived from the synchronous electromagnetic field which acts on the armature. Larmor's conjecture is that the self-excitation of the Sun establishes the orbital speed of the electromagnetic field, which determines the no load frequency of the armature. The rated speed of the Sun's armature will be somewhat less when fully loaded. This difference in synchronous and rated speed is commonly called slip. I currently do not know the fully loaded rated speed for a hydrogen gas machine like the Sun but will consider this for a future paper. Since the armature is not thought to be iron this will be a technical challenge. But, using orbital velocity of the electromagnetic field I have calculated an ideal frequency of $0.0011 \mathrm{~Hz}$ and a synchronous speed of $0.06696 \mathrm{RPM}$. This establishes a centripetal acceleration of $6709 \mathrm{~m} / \mathrm{s}^{2}$ at the surface of the Sun's inner core. Adjusting for the distance or altitude from the inner core to the surface of the Sun, I obtain a result of $268 \mathrm{~m} / \mathrm{s}^{2}$ which nearly matches the known value of $274 \mathrm{~m} / \mathrm{s}^{2}$ [11] -the error is less than $1 \%$.

The Standard Gravitation Parameter has units of $\mathrm{m}^{3} / \mathrm{s}^{2}$, which I interpret to mean acceleration of cubic volume of the solar system. The Sun by virtue of its constantly accelerating electromagnetic field is expanding the size of the solar system and pushing the planets outward by way of a centrifugal acceleration, or centrifugal force. Counter to the centrifugal force is the centripetal force, which is derived from the cross-product of the mass of the planets and the centripetal acceleration. The centripetal acceleration is a result of the rotating electromagnetic field reaching just beyond the planets and forcing the planets towards the Sun's center. Orbits of the planets around the Sun are the result of this balancing of centrifugal and centripetal forces, or gravity [12]. However in a two-body rotation, such as the Earth and moon rotating about their barycenter, the forces on both bodies are centripetal. In this case, the reaction to the centripetal force of 
Table 2. Comparing centripetal acceleration of the planets and Pluto with Newton's Law of Universal Gravitation.

\begin{tabular}{ccccccc}
\hline Planet & $\begin{array}{c}\text { Newton Law } \\
\text { Force }(\mathrm{N})\end{array}$ & $\begin{array}{c}\text { Mass } \\
(\mathrm{Kg})\end{array}$ & $\begin{array}{c}\text { Velocity } \\
(\mathrm{m} / \mathrm{s})\end{array}$ & $\begin{array}{c}\text { Distance } \\
(\mathrm{m})\end{array}$ & $\begin{array}{c}\text { Centripetal } \\
\text { Force }(\mathrm{N})\end{array}$ & $\begin{array}{c}\text { Error } \\
(\%)\end{array}$ \\
\hline Mercury & $1.31 \times 10^{22}$ & $3.30 \times 10^{23}$ & 47,400 & $5.79 \times 10^{10}$ & $1.28 \times 10^{22}$ & $0.02 \%$ \\
Venus & $5.54 \times 10^{22}$ & $4.87 \times 10^{24}$ & 35,000 & $1.08 \times 10^{11}$ & $5.52 \times 10^{22}$ & $0.26 \%$ \\
Earth & $3.54 \times 10^{22}$ & $5.98 \times 10^{24}$ & 29,800 & $1.50 \times 10^{11}$ & $3.54 \times 10^{22}$ & $0.03 \%$ \\
Mars & $1.64 \times 10^{21}$ & $6.42 \times 10^{23}$ & 24,000 & $2.28 \times 10^{11}$ & $1.62 \times 10^{22}$ & $1.01 \%$ \\
Jupiter & $4.16 \times 10^{23}$ & $1.90 \times 10^{27}$ & 13,000 & $7.78 \times 10^{11}$ & $4.12 \times 10^{23}$ & $0.94 \%$ \\
Saturn & $3.69 \times 10^{22}$ & $5.68 \times 10^{26}$ & 9700 & $1.43 \times 10^{12}$ & $3.73 \times 10^{22}$ & $1.05 \%$ \\
Uranus & $1.40 \times 10^{21}$ & $8.68 \times 10^{25}$ & 6800 & $2.87 \times 10^{12}$ & $1.40 \times 10^{21}$ & $0.09 \%$ \\
Neptune & $6.68 \times 10^{20}$ & $1.02 \times 10^{26}$ & 5400 & $4.50 \times 10^{12}$ & $6.62 \times 10^{20}$ & $1.03 \%$ \\
Pluto & $5.55 \times 10^{16}$ & $1.46 \times 10^{22}$ & 4700 & $5.91 \times 10^{12}$ & $5.46 \times 10^{16}$ & $1.59 \%$ \\
\hline
\end{tabular}

the Earth on the Moon is the centripetal force of the Moon on the Earth [13].

It is possible that the expansion and contraction of the universe is the result of the summation of all the stars and planets of the universe simultaneously performing this balancing act with their respective planets. If the universe is expanding, then the total centrifugal force is dominating the total centripetal force of the universe. It is thought that additional research into the velocity and acceleration of the Sun's electromagnetic field may help to understand the question regarding the expansion of the universe. It may be that young stars accelerate the universe and old stars decelerate.

The standard motor equation includes a time quantity of 60 , which represents 60 seconds per minute. Assuming all stars and planets can be modeled as constant speed dynamo's using a standard motor speed equation, it is theoretically possible that nearby solar systems are synchronized by way of flux transfer events between stars. Universal time may not be absolute, but our solar system is a very accurate time piece, clocking minutes and seconds by the constant speed of the Sun.

I conclude that gravity $(g)$ and Gravity $(G)$ are centripetal accelerations derived from a rotating electromagnetic field. To be recognized as a force of the universe there must be a dedicated field. What we have long called gravity has no such field. Electromagnetism alone transmits energy between matter in the cosmos. The Sun acts a cosmic power generator transmitting low frequency electrical energy to planets and maintaining their orbits by its powerful rotating magnetic field. There are only three known forces in the universe: weak nuclear, strong nuclear and electromagnetism.

\section{Acknowledgements}

The author wishes to acknowledge ASK Scientific (https://www.askscientific.com) for the formatting assistance and artwork. A special thanks to Dr. Christian Corda for allowing me the opportunity to publish technical papers that may 
someday open a door for theoretical power engineering in space. Cosmic power generation and transmission is a new frontier and Dr. Christian Corda was the first to recognize the possibilities.

\section{Conflicts of Interest}

The author declares no conflicts of interest regarding the publication of this paper.

\section{References}

[1] Larmor, S.J. (1919) How Could a Rotating Body Such as the Sun Become a Magnet? Report of the British Association for the Advancement of Science 87 th Meeting, 159-160.

[2] Gubbins, D. (2007) Larmor, Joseph (1857-1942). In: Gubbins, D. and Herrero-Bervera, E., Eds., Encyclopedia of Geomagnetism and Paleomagnetism, Springer, Dordrecht. https://doi.org/10.1007/978-1-4020-4423-6

[3] Fetter, A. and Walecka, J. (2003) Theoretical Mechanics of Particles and Continua. Dover Publications, Mineola, 13-22.

[4] García, R., Turck-Chièze, S., Jiménez-Reyes, S., Ballot, J., et al. (2007) Tracking Solar Gravity Modes: The Dynamics of the Solar Core. Science, 316, 1591-1593. https://doi.org/10.1126/science.1140598

[5] Raymond, H.M. (1910) Cyclopedia of Mechanical Engineering: A General Reference Work on Machine Shop... Volume 3, Chicago American Technical Society, Chicago, 10.

[6] Brumbach, M.E. and Clade, J. (2003) Industrial Maintenance. Thomson Delmar Learning, Library of Congress, Clifton Park, 400.

[7] Breithaupt, J. (2004) Physics for OCR. Nelson Thomas Ltd., Nashville.

[8] Calculated Gravity Acceleration. https://www.vcalc.com/wiki/KurtHeckman/Gravity+Acceleration+by+Altitude

[9] Milosevic, M. (2012) Internal Reflection and Spectroscopy. John Wiley \& Sons, Hoboken, 33.

[10] Poole, G. (2018) Cosmic Wireless Power Transfer System and the Equation for Everything $\mathrm{E}=\mathrm{mc}^{2}=\mathrm{vc}^{2} / 60=\mathrm{a}^{3} / \mathrm{T}=\mathrm{G}\left(\mathrm{M}_{1}+\mathrm{M}_{2}\right) / 4 \pi^{2}=(\mathrm{KE}+\mathrm{PE}) / 1.0 \mathrm{E} 15=\mathrm{Q}=$ $\mathrm{PA} / \mathrm{F}=\lambda / \mathrm{hc}=1 / 2 \mathrm{q}=\mathrm{VI}=1 / 2 \mathrm{LI}^{2}=1 / 2 \mathrm{CV}=\mathrm{I}^{2} \mathrm{R}=\ldots$. Journal of High Energy Physics, Gravitation and Cosmology, 4, 588-650. https://doi.org/10.4236/jhepgc.2018.44036

[11] McDougal, D.W. (2012) Newton's Gravity: An Introductory Guide to the Mechanics of the Universe. Springer and Associates, Berlin, 188.

[12] Corriveau, J.P. (2009) A Personal Journey into the Quantum World, God's Silent World. Universe Books, New York, 205.

[13] Scott, G.D. (1957) Centrifugal Forces and Newton's Laws of Motion. American Journal of Physics, 25, 586. https://doi.org/10.1119/1.1934450 